\title{
Prevention of the adverse effects of androgen deprivation therapy for prostate cancer using a 12- month home-based progressive resistance training program: a randomised controlled study
}

Teresa Lam ( $\sim$ Teresa.Lam@almedical.com.au )

Westmead Hospital

Birinder Cheema

Western Sydney University - Penrith Campus

Amy Hayden

Westmead Hospital

Stephen R. Lord

University of New South Wales

Howard Gurney

Westmead Hospital

Shivanjini Gounden

Western Sydney University

Navneeta Reddy

Blacktown Mount Druitt Hospital

Haleh Shahidipour

Blacktown Mount Druitt Hospital

Scott Read

Western Sydney University

Glenn Stone

Western Sydney University

Mark McLean

Blacktown Mount Druitt Hospital

Vita Birzniece

University of New South Wales

\section{Research Article}

Keywords: thyroid, PD-1, CTLA-4, immune reactions

Posted Date: January 10th, 2020 
DOI: https://doi.org/10.21203/rs.2.19495/v2

License: (c) (1) This work is licensed under a Creative Commons Attribution 4.0 International License. Read Full License 
The authors have withdrawn this preprint from Research Square 